\title{
Existence results of nonlocal boundary value problem for a nonlinear fractional differential coupled system involving fractional order impulses
}

\author{
Kaihong Zhao ${ }^{1 *}$ and Hui Huang ${ }^{1}$
}

\author{
"Correspondence: \\ zhaokaihongs@126.com \\ 'Department of Applied \\ Mathematics, Kunming University of \\ Science and Technology, Kunming, \\ China
}

\begin{abstract}
In this paper, we study the nonlocal boundary value problem for a nonlinear fractional differential coupled system with fractional order impulses. Applying Nonlinear Alternative of Leray-Schauder, we obtain some new existence results for this system. As application, an interesting example is given to illustrate the effectiveness of our main result.
\end{abstract}

MSC: 34B10; 34B15; 34B37

Keywords: Fractional differential coupled system; Nonlocal boundary value conditions; Impulses; Fixed point theorem

\section{Introduction}

In describing some phenomena and processes of many fields such as physics, chemistry, aerodynamics, electrodynamics of a complex medium, polymer rheology, capacitor theory, electrical circuits, biology, control theory, fitting of experimental data, and so on, the fractional order calculus is an excellent and more accurate tool than the integral order calculus. For example, in physics, we use Newtons' law $\eta \varepsilon^{\prime}(t)=\sigma(t)$ to describe the mechanics of viscous fluids, where $\sigma(t)$ and $\varepsilon(t)$ denote stress and strain at time $t$, respectively, and $\eta$ is the viscosity of the material. However, we need to employ Nuttings' law [1] $\eta D_{0^{+}}^{k} \varepsilon^{\prime}(t)=\sigma(t)(k \in(n-1, n), n \in \mathbb{N})$ to deal with the mechanics of viscous fluids involving some possible interpolation properties. As a consequence, the subject of fractional differential equations is gaining much importance and attention. There have been many papers focused on boundary value problems of fractional ordinary differential equations (see [131]). Especially, the nonlocal boundary value problems have been widely studied by many scholars because of their extensive applications in, e.g., blood flow problems, chemical engineering, thermo-elasticity, underground water flow, population dynamics, and so forth. The nonlocal boundary value problems of fractional-order differential equations constitute a class of very interesting and important problems. Such boundary value problems have been investigated in $[8-14,24,25,30]$.

In addition, the theory of impulse differential equations has seen significant development in recent years and played a very important role in modern applied mathematical

(c) The Author(s) 2019. This article is distributed under the terms of the Creative Commons Attribution 4.0 International License (http://creativecommons.org/licenses/by/4.0/), which permits unrestricted use, distribution, and reproduction in any medium, provided you give appropriate credit to the original author(s) and the source, provide a link to the Creative Commons license, and indicate if changes were made. 
models of real processes arising in phenomena studied in physics, population dynamics, chemical technology and biotechnology. Recently, some scholars have begun to study the boundary value problems for impulsive fractional differential equations (see [1, 15-26, 30, 32]). As is well known, the study on fractional differential coupled systems is more complicated and challenged than the study on a single fractional differential equation. Recently, some scholars began to investigate fractional differential coupled systems and obtained some good results (see $[8,12,13,24,26,31])$. However, there are few papers on the impulsive fractional order coupled systems with nonlocal boundary conditions and impulses. Therefore, in this paper, we consider the following four-point boundary value problem for nonlinear fractional differential coupling system with fractional order impulses of the form

$$
\left\{\begin{array}{l}
{ }^{C} D_{0^{+}}^{\alpha} x(t)=f\left(t, x(t),{ }^{C} D_{0^{+}}^{p} y(t)\right), \quad t \in J, t \neq t_{k}, \\
{ }^{C} D_{0^{+}}^{\beta} y(t)=g\left(t,{ }^{C} D_{0^{+}}^{q} x(t), y(t)\right), \quad t \in J, t \neq t_{k}, \\
{ }^{C} D_{0^{+}}^{\gamma_{1}} x\left(t_{k}^{+}\right)-{ }^{C} D_{0^{+}}^{\gamma_{1}} x\left(t_{k}^{-}\right)=J_{1 k}\left(x\left(t_{k}\right)\right), \quad k=1, \ldots, n, \\
{ }^{C} D_{0^{+}}^{\gamma_{2}} y\left(t_{k}^{+}\right)-{ }^{C} D_{0^{+}}^{\gamma_{2}} y\left(t_{k}^{-}\right)=J_{2 k}\left(y\left(t_{k}\right)\right), \quad k=1, \ldots, n, \\
x(0)=y(0)=0, \quad{ }^{\mathrm{LR}} D_{0^{+}}^{\delta_{1}} x(z)=x(1), \quad{ }^{\mathrm{LR}} D_{0^{+}}^{\delta_{2}} y(w)=y(1),
\end{array}\right.
$$

where $J=[0,1], 1<\alpha, \beta<2,0<p, q, \gamma_{1}, \gamma_{2}, \delta_{1}, \delta_{2}, z, w<1,{ }^{C} D_{0^{+}}^{\alpha},{ }^{C} D_{0^{+}}^{\beta},{ }^{C} D_{0^{+}}^{p},{ }^{C} D_{0^{+}}^{q}$, ${ }^{C} D_{0^{+}}^{\gamma_{1}}$, and ${ }^{C} D_{0^{+}}^{\gamma_{2}}$ are the Caputo fractional derivatives; ${ }^{\mathrm{LR}} D_{0^{+}}^{\delta_{1}}$ and ${ }^{\mathrm{LR}} D_{0^{+}}^{\delta_{2}}$ are the RiemannLiouville fractional derivatives; $f, g \in C\left(J \times R^{2}, R\right), J_{1 k}, J_{2 k} \in C(R, R)$, and $\left\{t_{k}\right\}$ satisfies $0=$ $t_{0}<t_{1}<\cdots<t_{n}<t_{n+1}=1,{ }^{C} D_{0^{+}}^{\gamma_{1}} x\left(t_{k}^{+}\right),{ }^{C} D_{0^{+}}^{\gamma_{1}} x\left(t_{k}^{-}\right),{ }^{C} D_{0^{+}}^{\gamma_{2}} y\left(t_{k}^{+}\right),{ }^{C} D_{0^{+}}^{\gamma_{2}} y\left(t_{k}^{-}\right)$all exist, ${ }^{C} D_{0^{+}}^{\gamma_{1}} x\left(t_{k}^{-}\right)=$ ${ }^{C} D_{0^{+}}^{\gamma_{1}} x\left(t_{k}\right),{ }^{C} D_{0^{+}}^{\gamma_{2}} y\left(t_{k}^{-}\right)={ }^{C} D_{0^{+}}^{\gamma_{1}} y\left(t_{k}\right), k=1,2, \ldots, n$.

The rest of this paper is organized as follows. In Sect. 2, we recall some definitions and lemmas of the Caputo and Riemann-Liouville fractional calculus. In Sect. 3, we shall prove the existence of solutions for system (1.1). In Sect. 4, some examples are given to demonstrate the application of our main results. Finally, conclusions are given in Sect. 5 to simply recall our studies and results obtained.

\section{Preliminaries}

Let $C(J)$ be the Banach space of continuous functions from $J$ to $\mathbb{R}$ with the norm $\|\psi\|_{C}=$ $\sup _{t \in J}|\psi(t)|$. Define the function space $\operatorname{PC}(J)$ by

$$
\begin{aligned}
\mathrm{PC}(J)= & \left\{\psi(t): \psi(t),{ }^{C} D_{0^{+}}^{p} \psi(t),{ }^{C} D_{0^{+}}^{q} \psi(t),{ }^{\mathrm{LR}} D_{0^{+}}^{\delta_{1}} \psi(t),{ }^{\mathrm{LR}} D_{0^{+}}^{\delta_{2}} \psi(t) \in C(J),{ }^{C} D_{0^{+}}^{\gamma_{1}} \psi\left(t_{k}^{+}\right),\right. \\
& { }^{C} D_{0^{+}}^{\gamma_{1}} \psi\left(t_{k}^{-}\right),{ }^{C} D_{0^{+}}^{\gamma_{2}} \psi\left(t_{k}^{+}\right) \text {and }{ }^{C} D_{0^{+}}^{\gamma_{2}} \psi\left(t_{k}^{-}\right) \text {all exist, and satisfy } \\
& { }^{C} D_{0^{+}}^{\gamma_{1}} \psi\left(t_{k}^{-}\right)={ }^{C} D_{0^{+}}^{\gamma_{1}} \psi\left(t_{k}\right),{ }^{C} D_{0^{+}}^{\gamma_{2}} \psi\left(t_{k}^{-}\right)={ }^{C} D_{0^{+}}^{\gamma_{2}} \psi\left(t_{k}\right), \\
& \left.0<p, q, \delta_{1}, \delta_{2}, \gamma_{1}, \gamma_{2}<1,1 \leq k \leq n\right\} .
\end{aligned}
$$

Obviously, $\mathrm{PC}(J)$ is a real Banach space equipped with the norm

$$
\|\psi\|_{\mathrm{PC}}=\max \left\{\|\psi\|_{C},\left\|^{c} D_{0^{+}}^{p} \psi\right\|_{C^{\prime}}\left\|^{c} D_{0^{+}}^{q} \psi\right\|_{C}\right\}, \quad \forall \psi \in \mathrm{PC}(J) .
$$

Let $X=\mathrm{PC}(J) \times \mathrm{PC}(J)$. It is easily to verify that $X$ is a Banach space with the norm $\|(u, v)\|=$ $\max \left\{\|u\|_{\mathrm{PC}},\|v\|_{\mathrm{PC}}\right\},(u, v) \in X$. 
For the readers' convenience, we introduce some necessary definitions and lemmas. These definitions and properties can be found in the literature.

Definition 2.1 ([32,33]) The Riemann-Liouville fractional integral of order $\alpha>0$ of a continuous function $f:(a, \infty) \rightarrow R$ is defined by

$$
I_{a^{+}}^{\alpha} f(t)=\frac{1}{\Gamma(\alpha)} \int_{a}^{t}(t-s)^{\alpha-1} f(s) d s
$$

provided that the right-hand side is pointwise defined on $(a, \infty)$.

Definition 2.2 ([33]) The Riemann-Liouville fractional derivative of order $\alpha>0$ of a continuous function $f:(a, \infty) \rightarrow R$ is defined by

$$
{ }^{\mathrm{LR}} D_{a^{+}}^{\alpha} f(t)=\frac{1}{\Gamma(n-\alpha)} \frac{d^{n}}{d t^{n}} \int_{0}^{t}(t-s)^{n-\alpha-1} f(s) d s,
$$

where $n-1<\alpha \leq n$, provided that the right-hand side is pointwise defined on $(a, \infty)$.

Definition 2.3 ([32, 33]) If $f \in C^{n}((a, \infty), R)$ and $\alpha>0$, then the Caputo fractional derivative of order $\alpha$ is defined as

$$
{ }^{C} D_{a^{+}}^{\alpha} f(t)=\frac{1}{\Gamma(n-\alpha)} \int_{a}^{t}(t-s)^{n-\alpha-1} f^{(n)}(s) d s,
$$

where $n-1<\alpha \leq n$, provided that the right-hand side is pointwise defined on $(a, \infty)$.

Lemma 2.1 ([33]) If $u \in C^{n}[0,1]$, and $\delta>0$, then

$$
I_{0^{+}}^{\delta}{ }^{C} D_{0^{+}}^{\delta} u(t)=u(t)-\sum_{k=0}^{n-1} \frac{u^{(k)}(0)}{k !} t^{k},
$$

where $n=-[-\delta]$ and $[-\delta]$ denotes the integer part of the real number $-\delta$.

Lemma $2.2([32,33])$ If $\alpha, \beta>0, t \in[a, b]$ and $u(t) \in L[a, b]$, then

$$
{ }^{C} D_{a^{+}}^{\alpha} I_{a^{+}}^{\alpha} u(t)=u(t), \quad I_{a^{+}}^{\alpha} a_{a^{+}}^{\beta} u(t)=I_{a^{+}}^{\alpha+\beta} u(t) .
$$

Lemma 2.3 (see [34], pp. 36-39) Let $\alpha>0$ and suppose $n$ denotes the smallest integer greater than or equal to $\alpha$. Then the following assertions hold:

(i) If $\lambda>-1, \lambda \neq \alpha-i, i=1,2, \ldots, n+1$, then for $t \in[a, b]$,

$$
{ }^{\mathrm{LR}} D_{a^{+}}^{\alpha}(t-a)^{\lambda}=\frac{\Gamma(\lambda+1)}{\Gamma(\lambda-\alpha+1)}(t-a)^{\lambda-\alpha} .
$$

(ii) ${ }^{\mathrm{LR}} D_{a^{+}}^{\alpha}(t-a)^{\alpha-i}=0, i=1,2, \ldots, n$.

(iii) ${ }^{\mathrm{LR}} D_{a^{+}}^{\beta} I_{a^{+}}^{\alpha} u(t)=I_{a^{+}}^{\alpha} u(t)$, for all $t \in[a, b], \alpha \geq \beta \geq 0$.

Lemma 2.4 (Nonlinear Alternative Of Leray-Schauder [35]) Let $X$ be a Banach space, $C$ be a nonempty convex subset of $X, \Omega$ be an open subset of $C$ with $\theta \in \Omega$. Suppose that $T: \bar{\Omega} \rightarrow C$ is a completely continuous mapping. Then either 
(i) the mapping $T$ has a fixed point in $\bar{\Omega}$, or

(ii) there exists $a u \in \partial \Omega$ and $\lambda \in(0,1)$ with $u=\lambda T u$.

Lemma 2.5 Let $h_{1} \in C(J)$. If $\Delta_{1} \triangleq \frac{z^{1-\delta_{1}}}{\Gamma\left(2-\delta_{1}\right)} \neq 1$, then a function $x \in \mathrm{PC}(J)$ is a solution of the boundary value problem

$$
\left\{\begin{array}{l}
{ }^{C} D_{0^{+}}^{\alpha} x(t)=h_{1}(t), \quad 1<\alpha<2, t \in J, t \neq t_{k}, \\
{ }^{C} D_{0^{+}}^{\gamma_{1}} x\left(t_{k}^{+}\right)-{ }^{C} D_{0^{+}}^{\gamma_{1}} x\left(t_{k}^{-}\right)=J_{1 k}\left(x\left(t_{k}\right)\right), \quad 0<\gamma_{1}<1, k=1,2, \ldots, n, \\
x(0)=0, \quad{ }^{\mathrm{LR}} D_{0^{+}}^{\delta_{1}} x(z)=x(1), \quad 0<\delta_{1}<1, \quad 0<z<1,
\end{array}\right.
$$

if and only if $x \in \mathrm{PC}(J)$ is a solution of the integral equation

$$
\begin{aligned}
x(t)= & I_{0^{+}}^{\alpha} h_{1}(t)+\left(\frac{I_{0^{+}}^{\alpha-\delta_{1}} h_{1}(z)-I_{0^{+}}^{\alpha} h_{1}(1)+C_{1}}{1-\Delta_{1}}+\sum_{i=1}^{k} \frac{\Gamma\left(2-\gamma_{1}\right)}{t_{i}^{1-\gamma_{1}}} J_{1 i}\left(x\left(t_{i}\right)\right)\right) t \\
& -\Gamma\left(2-\gamma_{1}\right) t_{k}^{\gamma_{1}} J_{1 k}\left(x\left(t_{k}\right)\right), \quad t \in\left(t_{k}, t_{k+1}\right], k=0,1, \ldots, n,
\end{aligned}
$$

where

$$
\begin{aligned}
C_{1}= & \Delta_{1} \Gamma\left(2-\gamma_{1}\right) \sum_{i=1}^{j} \frac{J_{1 i}\left(x\left(t_{i}\right)\right)}{t_{i}^{1-\gamma_{1}}}-\frac{z^{-\delta_{1}} t_{j}^{\gamma_{1}} \Gamma\left(2-\gamma_{1}\right)}{\Gamma\left(1-\delta_{1}\right)} J_{1 j}\left(x\left(t_{j}\right)\right)+t_{n}^{\gamma_{1}} \Gamma\left(2-\gamma_{1}\right) J_{1 n}\left(x\left(t_{n}\right)\right) \\
& -\Gamma\left(2-\gamma_{1}\right) \sum_{i=1}^{n} \frac{J_{1 i}\left(x\left(t_{i}\right)\right)}{t_{i}^{1-\gamma_{1}}}, \quad t_{j}<z \leq t_{j+1}, j \in\{0,1,2, \ldots, n\} .
\end{aligned}
$$

Proof When $t \in\left[0, t_{1}\right]$, from Lemma 2.1, we have

$$
x(t)=I_{0^{+}}^{\alpha} h_{1}(t)+u_{10}+u_{11} t=\frac{1}{\Gamma(\alpha)} \int_{0}^{t}(t-s)^{\alpha-1} h_{1}(s) d s+u_{10}+u_{11} t .
$$

By $x(0)=0$, we get $u_{10}=0$. And it follows from (2.3) that

$$
{ }^{C} D_{0^{+}}^{\gamma_{1}} x(t)=\frac{u_{11} t^{1-\gamma_{1}}}{\Gamma\left(2-\gamma_{1}\right)}+\frac{1}{\Gamma\left(\alpha-\gamma_{1}\right)} \int_{0}^{t}(t-s)^{\alpha-\gamma_{1}-1} h_{1}(s) d s,
$$

and

$$
{ }^{C} D_{0^{+}}^{\gamma_{1}} x\left(t_{1}^{-}\right)={ }^{C} D_{0^{+}}^{\gamma_{1}} x\left(t_{1}\right)=\frac{u_{11} t_{1}^{1-\gamma_{1}}}{\Gamma\left(2-\gamma_{1}\right)}+\frac{1}{\Gamma\left(\alpha-\gamma_{1}\right)} \int_{0}^{t_{1}}\left(t_{1}-s\right)^{\alpha-\gamma_{1}-1} h_{1}(s) d s .
$$

When $t \in\left(t_{1}, t_{2}\right]$, we similarly have

$$
\begin{aligned}
& x(t)=I_{0^{+}}^{\alpha} h_{1}(t)+u_{20}+u_{21} t=\frac{1}{\Gamma(\alpha)} \int_{0}^{t}(t-s)^{\alpha-1} h_{1}(s) d s+u_{20}+u_{21} t, \\
& { }^{C} D_{0^{+}}^{\gamma_{1}} x(t)=\frac{u_{21} t^{1-\gamma_{1}}}{\Gamma\left(2-\gamma_{1}\right)}+\frac{1}{\Gamma\left(\alpha-\gamma_{1}\right)} \int_{0}^{t}(t-s)^{\alpha-\gamma_{1}-1} h_{1}(s) d s,
\end{aligned}
$$

and

$$
{ }^{C} D_{0^{+}}^{\gamma_{1}} x\left(t_{1}^{+}\right)=\frac{u_{21} t_{1}^{1-\gamma_{1}}}{\Gamma\left(2-\gamma_{1}\right)}+\frac{1}{\Gamma\left(\alpha-\gamma_{1}\right)} \int_{0}^{t_{1}}\left(t_{1}-s\right)^{\alpha-\gamma_{1}-1} h_{1}(s) d s .
$$


By (2.5), (2.8) and ${ }^{C} D_{0^{+}}^{\gamma_{1}} x\left(t_{k}^{+}\right)-{ }^{C} D_{0^{+}}^{\gamma_{1}} x\left(t_{k}^{-}\right)=J_{1 k}\left(x\left(t_{k}\right)\right)$, we obtain

$$
u_{21}-u_{11}=\frac{\Gamma\left(2-\gamma_{1}\right)}{t_{1}^{1-\gamma_{1}}} J_{11}\left(x\left(t_{1}\right)\right)
$$

In view of the continuity of $x$ at $t_{1}$, we have

$$
u_{20}=-\left(u_{21}-u_{11}\right) t_{1}=-\Gamma\left(2-\gamma_{1}\right) t_{1}^{\gamma_{1}} J_{11}\left(x\left(t_{1}\right)\right) \text {. }
$$

When $t \in\left(t_{k}, t_{k+1}\right], k=2,3, \ldots, n$, repeating the above calculation, we get

$$
x(t)=\frac{1}{\Gamma(\alpha)} \int_{0}^{t}(t-s)^{\alpha-1} h_{1}(s) d s+u_{k+1,0}+u_{k+1,1} t
$$

and

$$
u_{k+1,1}-u_{k 1}=\frac{\Gamma\left(2-\gamma_{1}\right)}{t_{k}^{1-\gamma_{1}}} J_{1 k}\left(x\left(t_{k}\right)\right), \quad u_{k+1,0}=-\Gamma\left(2-\gamma_{1}\right) t_{k}^{\gamma_{1}} J_{1 k}\left(x\left(t_{k}\right)\right) .
$$

From (2.11) and (2.12), we have

$$
x(1)=\frac{1}{\Gamma(\alpha)} \int_{0}^{1}(1-s)^{\alpha-1} h_{1}(s) d s-\Gamma\left(2-\gamma_{1}\right) t_{n}^{\gamma_{1}} J_{1 n}\left(x\left(t_{n}\right)\right)+u_{n+1,1} .
$$

Equations (2.9) and (2.12) give

$$
u_{k+1,1}=u_{11}+\sum_{i=1}^{k} \frac{\Gamma\left(2-\gamma_{1}\right)}{t_{i}^{1-\gamma_{1}}} J_{1 i}\left(x\left(t_{i}\right)\right), \quad k=1,2, \ldots, n
$$

Denoting $t_{0}=0, t_{n+1}=1$, and noticing $0<z<1$, we know that there exists $j \in\{0,1, \ldots, n\}$ such that $z \in\left(t_{j}, t_{j+1}\right]$ and

$$
x(z)=\frac{1}{\Gamma(\alpha)} \int_{0}^{z}(z-s)^{\alpha-1} h_{1}(s) d s+u_{j+1,0}+u_{j+1,1} z=I_{0^{+}}^{\alpha} h_{1}(z)+u_{j+1,0}+u_{j+1,1} z .
$$

Applying Lemmas 2.2-2.3 and (2.15), we obtain

$$
{ }^{\mathrm{LR}} D_{0^{+}}^{\delta_{1}} x(z)=\frac{1}{\Gamma\left(\alpha-\delta_{1}\right)} \int_{0}^{z}(z-s)^{\alpha-\delta_{1}-1} h_{1}(s) d s+\frac{u_{j+1,1} \Gamma(2) z^{1-\delta_{1}}}{\Gamma\left(2-\delta_{1}\right)}+\frac{u_{j+1,0} z^{-\delta_{1}}}{\Gamma\left(1-\delta_{1}\right)}
$$

Using ${ }^{\mathrm{LR}} D_{0^{+}}^{\delta_{1}} x(z)=x(1),(2.12),(2.13),(2.14)$ and (2.16), we derive

$$
\begin{aligned}
u_{11}= & \frac{1}{\left(1-\Delta_{1}\right) \Gamma\left(\alpha-\delta_{1}\right)} \int_{0}^{z}(z-s)^{\alpha-\delta_{1}-1} h_{1}(s) d s+\frac{\Delta_{1} \Gamma\left(2-\gamma_{1}\right)}{1-\Delta_{1}} \sum_{i=1}^{j} \frac{J_{1 i}\left(x\left(t_{i}\right)\right)}{t_{i}^{1-\gamma_{1}}} \\
& -\frac{z^{-\delta_{1}} t_{j}^{\gamma_{1}} \Gamma\left(2-\gamma_{1}\right)}{\left(1-\Delta_{1}\right) \Gamma\left(1-\delta_{1}\right)} J_{1 j}\left(x\left(t_{j}\right)\right)-\frac{1}{\left(1-\Delta_{1}\right) \Gamma(\alpha)} \int_{0}^{1}(1-s)^{\alpha-1} h_{1}(s) d s \\
& +\frac{t_{n}^{\gamma_{1}} \Gamma\left(2-\gamma_{1}\right)}{1-\Delta_{1}} J_{1 n}\left(x\left(t_{n}\right)\right)-\frac{\Gamma\left(2-\gamma_{1}\right)}{1-\Delta_{1}} \sum_{i=1}^{n} \frac{J_{1 i}\left(x\left(t_{i}\right)\right)}{t_{i}^{1-\gamma_{1}}}
\end{aligned}
$$




$$
\begin{aligned}
= & \frac{1}{1-\Delta_{1}}\left[I_{0^{+}}^{\alpha-\delta_{1}} h_{1}(z)-I_{0^{+}}^{\alpha} h_{1}(1)+\Delta_{1} \Gamma\left(2-\gamma_{1}\right) \sum_{i=1}^{j} \frac{J_{1 i}\left(x\left(t_{i}\right)\right)}{t_{i}^{1-\gamma_{1}}}-\frac{z^{-\delta_{1}} t_{j}^{\gamma_{1}} \Gamma\left(2-\gamma_{1}\right)}{\Gamma\left(1-\delta_{1}\right)}\right. \\
& \left.\times J_{1 j}\left(x\left(t_{j}\right)\right)+t_{n}^{\gamma_{1}} \Gamma\left(2-\gamma_{1}\right) J_{1 n}\left(x\left(t_{n}\right)\right)-\Gamma\left(2-\gamma_{1}\right) \sum_{i=1}^{n} \frac{J_{1 i}\left(x\left(t_{i}\right)\right)}{t_{i}^{1-\gamma_{1}}}\right] \\
= & \frac{A_{1}}{1-\Delta_{1}} .
\end{aligned}
$$

Thus, for $\left(t_{k}, t_{k+1}\right], k=0,1,2, \ldots, n$, we have

$$
\begin{aligned}
x(t) & =\frac{1}{\Gamma(\alpha)} \int_{0}^{t}(t-s)^{\alpha-1} h_{1}(s) d s+u_{k+1,0}+u_{k+1,1} t \\
& =I_{0^{+}}^{\alpha} h_{1}(t)-\Gamma\left(2-\gamma_{1}\right) t_{k}^{\gamma_{1}} J_{1 k}\left(x\left(t_{k}\right)\right)+\left(u_{11}+\sum_{i=1}^{k} \frac{\Gamma\left(2-\gamma_{1}\right)}{t_{i}^{1-\gamma_{1}}} J_{1 i}\left(x\left(t_{i}\right)\right)\right) t .
\end{aligned}
$$

Substituting (2.17) into (2.18), one can easily obtain (2.2). The proof is completed.

Similarly, we conclude the following lemma.

Lemma 2.6 Let $h_{2} \in \mathrm{PC}(J)$. If $\Delta_{2} \triangleq \frac{w^{1-\delta_{2}}}{\Gamma\left(2-\delta_{2}\right)} \neq 1$, then a function $y \in \mathrm{PC}(J)$ is a solution of the boundary value problem

$$
\left\{\begin{array}{l}
{ }^{C} D_{0^{+}}^{\beta} y(t)=h_{2}(t), \quad 1<\beta<2, t \in J, t \neq t_{k}, \\
{ }^{C} D_{0^{+}}^{\gamma_{2}} y\left(t_{k}^{+}\right)-{ }^{C} D_{0^{+}}^{\gamma_{2}} y\left(t_{k}^{-}\right)=J_{2 k}\left(y\left(t_{k}\right)\right), \quad 0<\gamma_{2}<1, k=1,2, \ldots, n, \\
y(0)=0, \quad{ }^{\mathrm{LR}} D_{0^{+}}^{\delta_{2}} y(w)=y(1), \quad 0<\delta_{2}<1, \quad 0<w<1,
\end{array}\right.
$$

if and only if $y \in \mathrm{PC}(J)$ is a solution of the integral equation

$$
\begin{aligned}
x(t)= & I_{0^{+}}^{\beta} h_{2}(t)+\left(\frac{I_{0^{+}}^{\beta-\delta_{2}} h_{2}(w)-I_{0^{+}}^{\beta} h_{2}(1)+C_{2}}{1-\Delta_{2}}+\sum_{i=1}^{k} \frac{\Gamma\left(2-\gamma_{2}\right)}{t_{i}^{1-\gamma_{2}}} J_{2 i}\left(y\left(t_{i}\right)\right)\right) t \\
& -\Gamma\left(2-\gamma_{2}\right) t_{k}^{\gamma_{2}} J_{2 k}\left(y\left(t_{k}\right)\right), \quad t \in\left(t_{k}, t_{k+1}\right], k=0,1, \ldots, n,
\end{aligned}
$$

where

$$
\begin{aligned}
C_{2}= & \Delta_{2} \Gamma\left(2-\gamma_{2}\right) \sum_{i=1}^{l} \frac{J_{2 i}\left(y\left(t_{i}\right)\right)}{t_{i}^{1-\gamma_{2}}}-\frac{w^{-\delta_{2}} t_{l}^{\gamma_{2}} \Gamma\left(2-\gamma_{2}\right)}{\Gamma\left(1-\delta_{2}\right)} J_{2 l}\left(y\left(t_{l}\right)\right)+t_{n}^{\gamma_{2}} \Gamma\left(2-\gamma_{2}\right) J_{2 n}\left(y\left(t_{n}\right)\right) \\
& -\Gamma\left(2-\gamma_{2}\right) \sum_{i=1}^{n} \frac{J_{2 i}\left(y\left(t_{i}\right)\right)}{t_{i}^{1-\gamma_{2}}}, \quad t_{l}<w \leq t_{l+1}, l \in\{0,1,2, \ldots, n\} .
\end{aligned}
$$

\section{Main results}

In this section, we shall investigate the existence of solution for system (1.1) by employing the nonlinear alternative of Leray-Schauder.

Theorem 3.1 If the following conditions $\left(H_{1}\right)-\left(H_{6}\right)$ hold, then the boundary value problem (1.1) has at least a pair of solutions. The conditions are:

$\left(H_{1}\right)$ The functions $f, g \in C\left(J \times R^{2}, R\right)$, and $J_{1 k}, J_{2 k} \in C(R, R), k=1,2, \ldots, n$. 
$\left(H_{2}\right)$ For all $u_{i}, v_{i} \in R(i=1,2), t \in R$, there exist some constants $L_{i}, \hat{L}_{i}>0(i=1,2)$ such that

$$
\begin{aligned}
& \left|f\left(t, u_{1}, v_{1}\right)-f\left(t, u_{2}, v_{2}\right)\right| \leq L_{1}\left|u_{1}-u_{2}\right|+L_{2}\left|v_{1}-v_{2}\right|, \\
& \left|g\left(t, u_{1}, v_{1}\right)-g\left(t, u_{2}, v_{2}\right)\right| \leq \hat{L}_{1}\left|u_{1}-u_{2}\right|+\hat{L}_{2}\left|v_{1}-v_{2}\right| .
\end{aligned}
$$

$\left(H_{3}\right) N \triangleq \sup _{t \in[0,1]}|f(t, 0,0)|$ and $\hat{N} \triangleq \sup _{t \in[0,1]}|g(t, 0,0)|$ all exist.

$\left(H_{4}\right) 0<\Delta_{1}=\frac{z^{1-\delta_{1}}}{\Gamma\left(2-\delta_{1}\right)}<1,0<\Delta_{2}=\frac{w^{1-\delta_{2}}}{\Gamma\left(2-\delta_{2}\right)}<1$.

$\left(H_{5}\right)$ For any $u, v \in R$, there exist some constants $M_{k}, \hat{M}_{k}>0, k=1,2, \ldots, n$, such that

$$
\left|J_{1 k}(u)\right| \leq M_{k}|u|, \quad\left|J_{2 k}(v)\right| \leq \hat{M}_{k}|v| .
$$

$\left(H_{6}\right) \kappa_{1} \triangleq \mathcal{M}_{1}+\mathcal{N}_{1}<1$ and $\kappa_{2} \triangleq \mathcal{M}_{2}+\mathcal{N}_{2}<1$, where

$$
\begin{aligned}
& \mathcal{M}_{1}=\left(L_{1}+L_{2}\right)\left(\frac{1}{\Gamma(\alpha+1)}+\frac{1}{\left(1-\Delta_{1}\right) \Gamma\left(\alpha-\delta_{1}+1\right)}+\frac{1}{\left(1-\Delta_{1}\right) \Gamma(\alpha+1)}\right), \\
& \mathcal{N}_{1}=\frac{\Gamma\left(2-\gamma_{1}\right)}{1-\Delta_{1}}\left(\frac{2}{t_{1}^{1-\gamma_{1}}}+\frac{1}{z^{\delta_{1}} \Gamma\left(1-\delta_{1}\right)}+2-\Delta_{1}\right) \sum_{i=1}^{n} M_{i}, \\
& \mathcal{M}_{2}=\left(\hat{L}_{1}+\hat{L}_{2}\right)\left(\frac{1}{\Gamma(\beta+1)}+\frac{1}{\left(1-\Delta_{2}\right) \Gamma\left(\beta-\delta_{2}+1\right)}+\frac{1}{\left(1-\Delta_{2}\right) \Gamma(\beta+1)}\right), \\
& \mathcal{N}_{2}=\frac{\Gamma\left(2-\gamma_{2}\right)}{1-\Delta_{2}}\left(\frac{2}{t_{1}^{1-\gamma_{2}}}+\frac{1}{w^{\delta_{2}} \Gamma\left(1-\delta_{2}\right)}+2-\Delta_{2}\right) \sum_{i=1}^{n} \hat{M}_{i} .
\end{aligned}
$$

Proof Let $\Omega=\{(x, y) \in X:\|(x, y)\|<r\}$, where $X=\operatorname{PC}(J) \times \operatorname{PC}(J)$ and $r \geq \max \left\{\frac{N \mathcal{M}_{1}}{1-\kappa_{1}}, \frac{\hat{N} \mathcal{M}_{2}}{1-\kappa_{2}}\right\}$. Then $\bar{\Omega}=\{(x, y) \in X:\|(x, y)\| \leq r\}, \partial \Omega=\{(x, y) \in X:\|(x, y)\|=r\}$. According to Lemmas 2.5-2.6, we define the operator $T: \bar{\Omega} \rightarrow X$ as follows:

$$
T(x, y)(t)=\left(T_{1}(x, y)(t), T_{2}(x, y)(t)\right)^{T}, \quad \forall(x, y) \in X, t \in J
$$

where

$$
\begin{aligned}
T_{1}(x, y)(t)= & I_{0^{+}}^{\alpha} f\left(t, x(t),{ }^{C} D_{0^{+}}^{p} y(t)\right) \\
& +\frac{I_{0^{+}}^{\alpha-\delta_{1}} f\left(z, x(z),{ }^{C} D_{0^{+}}^{p} y(z)\right)-I_{0^{+}}^{\alpha} f\left(1, x(1),{ }^{C} D_{0^{+}}^{p} y(1)\right)}{1-\Delta_{1}} t \\
& +\left(\frac{C_{1}}{1-\Delta_{1}}+\sum_{i=1}^{k} \frac{\Gamma\left(2-\gamma_{1}\right)}{t_{i}^{1-\gamma_{1}}} J_{1 i}\left(x\left(t_{i}\right)\right)\right) t-\Gamma\left(2-\gamma_{1}\right) t_{k}^{\gamma_{1}} J_{1 k}\left(x\left(t_{k}\right)\right), \\
& t \in\left(t_{k}, t_{k+1}\right], k=0,1, \ldots, n, \\
T_{2}(x, y)(t)= & I_{0^{+}}^{\beta} g\left(t,{ }^{C} D_{0^{+}}^{q} x(t), y(t)\right) \\
& +\frac{I_{0^{+}}^{\beta-\delta_{2}} g\left(w,{ }^{C} D_{0^{+}}^{q} x(w), y(w)\right)-I_{0^{+}}^{\beta} g\left(1,{ }^{C} D_{0^{+}}^{q} x(1), y(1)\right)}{1-\Delta_{2}} t \\
& +\left(\frac{C_{2}}{1-\Delta_{2}}+\sum_{i=1}^{k} \frac{\Gamma\left(2-\gamma_{2}\right)}{t_{i}^{1-\gamma_{2}}} J_{2 i}\left(y\left(t_{i}\right)\right)\right) t-\Gamma\left(2-\gamma_{2}\right) t_{k}^{\gamma_{2}} J_{2 k}\left(y\left(t_{k}\right)\right), \\
& t \in\left(t_{k}, t_{k+1}\right], k=0,1, \ldots, n,
\end{aligned}
$$




$$
\begin{aligned}
C_{1}= & \Delta_{1} \Gamma\left(2-\gamma_{1}\right) \sum_{i=1}^{j} \frac{J_{1 i}\left(x\left(t_{i}\right)\right)}{t_{i}^{1-\gamma_{1}}}-\frac{z^{-\delta_{1}} t_{j}^{\gamma_{1}} \Gamma\left(2-\gamma_{1}\right)}{\Gamma\left(1-\delta_{1}\right)} J_{1 j}\left(x\left(t_{j}\right)\right) \\
& +t_{n}^{\gamma_{1}} \Gamma\left(2-\gamma_{1}\right) J_{1 n}\left(x\left(t_{n}\right)\right)-\Gamma\left(2-\gamma_{1}\right) \sum_{i=1}^{n} \frac{J_{1 i}\left(x\left(t_{i}\right)\right)}{t_{i}^{1-\gamma_{1}}} \\
& t_{j}<z \leq t_{j+1}, j \in\{0,1,2, \ldots, n\},
\end{aligned}
$$

and

$$
\begin{aligned}
C_{2}= & \Delta_{2} \Gamma\left(2-\gamma_{2}\right) \sum_{i=1}^{l} \frac{J_{2 i}\left(y\left(t_{i}\right)\right)}{t_{i}^{1-\gamma_{2}}}-\frac{w^{-\delta_{2}} t_{l}^{\gamma_{2}} \Gamma\left(2-\gamma_{2}\right)}{\Gamma\left(1-\delta_{2}\right)} J_{2 l}\left(y\left(t_{l}\right)\right) \\
& +t_{n}^{\gamma_{2}} \Gamma\left(2-\gamma_{2}\right) J_{2 n}\left(y\left(t_{n}\right)\right)-\Gamma\left(2-\gamma_{2}\right) \sum_{i=1}^{n} \frac{J_{2 i}\left(y\left(t_{i}\right)\right)}{t_{i}^{1-\gamma_{2}}} \\
& t_{l}<w \leq t_{l+1}, l \in\{0,1,2, \ldots, n\} .
\end{aligned}
$$

Thus, the existence of solution for system (1.1) is equivalent to the existence of a fixed point for the operator $T$ defined by (3.1)-(3.5). Now we shall apply Lemma 2.4 to prove that $T$ has a fixed point $\left(x^{*}(t), y^{*}(t)\right) \in \bar{\Omega}$. Firstly, we need to show that $T: \bar{\Omega} \rightarrow X$ is completely continuous. In fact, for all $(x, y) \in \bar{\Omega}, t \in J=[0,1]$, from conditions $\left(H_{1}\right)-\left(H_{5}\right)$, we have

$$
\begin{aligned}
\left|T_{1}(x, y)(t)\right| & \leq I_{0^{+}}^{\alpha}\left|f\left(t, x(t),{ }^{C} D_{0^{+}}^{p} y(t)\right)\right| \\
& +\frac{I_{0^{+}}^{\alpha-\delta_{1}}\left|f\left(z, x(z),{ }^{C} D_{0^{+}}^{p} y(z)\right)\right|+I_{0^{+}}^{\alpha}\left|f\left(1, x(1),{ }^{C} D_{0^{+}}^{p} y(1)\right)\right|}{1-\Delta_{1}} \\
& +\frac{\left|C_{1}\right|}{1-\Delta_{1}}+\sum_{i=1}^{k} \frac{\Gamma\left(2-\gamma_{1}\right)}{t_{i}^{1-\gamma_{1}}}\left|J_{1 i}\left(x\left(t_{i}\right)\right)\right|+\Gamma\left(2-\gamma_{1}\right) t_{k}^{\gamma_{1}}\left|J_{1 k}\left(x\left(t_{k}\right)\right)\right| \\
\leq & I_{0^{+}}^{\alpha}\left|f\left(t, x(t),{ }^{C} D_{0^{+}}^{p} y(t)\right)-f(t, 0,0)\right|+I_{0^{+}}^{\alpha}|f(t, 0,0)| \\
& +\frac{1}{1-\Delta_{1}}\left[I_{0^{+}}^{\alpha-\delta_{1}}\left|f\left(z, x(z),{ }^{C} D_{0^{+}}^{p} y(z)\right)-f(z, 0,0)\right|+I_{0^{+}}^{\alpha-\delta_{1}}|f(z, 0,0)|\right] \\
& +\frac{1}{1-\Delta_{1}}\left[I_{0^{+}}^{\alpha}\left|f\left(1, x(1),{ }^{C} D_{0^{+}}^{p} y(1)\right)-f(1,0,0)\right|+I_{0^{+}}^{\alpha}|f(1,0,0)|\right] \\
& +\frac{1}{1-\Delta_{1}}\left[\Delta_{1} \Gamma\left(2-\gamma_{1}\right) \sum_{i=1}^{j} \frac{\left|J_{1 i}\left(x\left(t_{i}\right)\right)\right|}{t_{i}^{1-\gamma_{1}}}+\frac{z^{-\delta_{1}} t_{j}^{\gamma_{1}} \Gamma\left(2-\gamma_{1}\right)}{\Gamma\left(1-\delta_{1}\right)}\left|J_{1 j}\left(x\left(t_{j}\right)\right)\right|\right. \\
& \left.+t_{n}^{\gamma_{1}} \Gamma\left(2-\gamma_{1}\right)\left|J_{1 n}\left(x\left(t_{n}\right)\right)\right|+\Gamma\left(2-\gamma_{1}\right) \sum_{i=1}^{n} \frac{\left|J_{1 i}\left(x\left(t_{i}\right)\right)\right|}{\left.t_{i}^{1-\gamma_{1}}\right]}\right] \\
& +\sum_{i=1}^{k} \frac{\Gamma\left(2-\gamma_{1}\right)}{t_{i}^{1-\gamma_{1}} \mid}\left|J_{1 i}\left(x\left(t_{i}\right)\right)\right|+\Gamma\left(2-\gamma_{1}\right) t_{k}^{\gamma_{1}}\left|J_{1 k}\left(x\left(t_{k}\right)\right)\right| \\
\leq & I_{0^{+}}^{\alpha}\left(L_{1}|x(t)|+L_{2}\left|{ }^{C} D_{0^{+}}^{p} y(t)\right|\right)+I_{0^{+}}^{\alpha} \sup _{0 \leq t \leq 1}|f(t, 0,0)|
\end{aligned}
$$




$$
\begin{aligned}
& \left.+\frac{1}{1-\Delta_{1}}\left[I_{0^{+}}^{\alpha-\delta_{1}}\left(L_{1}|x(z)|+L_{2} \mid{ }^{C} D_{0^{+}}^{p} y(z)\right) \mid\right)+I_{0^{+}}^{\alpha-\delta_{1}} \sup _{0 \leq t \leq 1}|f(t, 0,0)|\right] \\
& +\frac{1}{1-\Delta_{1}}\left[I_{0^{+}}^{\alpha}\left(L_{1}|x(1)|+L_{2}\left|{ }^{C} D_{0^{+}}^{p} y(1)\right|\right)+I_{0^{+}}^{\alpha} \sup _{0 \leq t \leq 1}|f(t, 0,0)|\right] \\
& +\frac{1}{1-\Delta_{1}}\left[\Delta_{1} \Gamma\left(2-\gamma_{1}\right) \sum_{i=1}^{j} \frac{M_{i}\left|x\left(t_{i}\right)\right|}{t_{i}^{1-\gamma_{1}}}+\frac{z^{-\delta_{1}} t_{j}^{\gamma_{1}} \Gamma\left(2-\gamma_{1}\right)}{\Gamma\left(1-\delta_{1}\right)} M_{j}\left|x\left(t_{j}\right)\right|\right. \\
& \left.+t_{n}^{\gamma_{1}} \Gamma\left(2-\gamma_{1}\right) M_{n}\left|x\left(t_{n}\right)\right|+\Gamma\left(2-\gamma_{1}\right) \sum_{i=1}^{n} \frac{M_{i}\left|x\left(t_{i}\right)\right|}{t_{i}^{1-\gamma_{1}}}\right] \\
& +\sum_{i=1}^{k} \frac{\Gamma\left(2-\gamma_{1}\right)}{t_{i}^{1-\gamma_{1}}} M_{i}\left|x\left(t_{i}\right)\right|+\Gamma\left(2-\gamma_{1}\right) t_{k}^{\gamma_{1}} M_{k}\left|x\left(t_{k}\right)\right| \\
& \leq \frac{1}{\Gamma(\alpha+1)}\left(L_{1}\|x\|_{\mathrm{PC}}+L_{2}\|y\|_{\mathrm{PC}}+N\right) \\
& +\frac{1}{\left(1-\Delta_{1}\right) \Gamma\left(\alpha-\delta_{1}+1\right)}\left(L_{1}\|x\|_{\mathrm{PC}}+L_{2}\|y\|_{\mathrm{PC}}+N\right) \\
& +\frac{1}{\left(1-\Delta_{1}\right) \Gamma(\alpha+1)}\left(L_{1}\|x\|_{\mathrm{PC}}+L_{2}\|y\|_{\mathrm{PC}}+N\right) \\
& +\frac{\Gamma\left(2-\gamma_{1}\right)}{1-\Delta_{1}}\left[\frac{2}{t_{1}^{1-\gamma_{1}}}+\frac{1}{z^{\delta_{1}} \Gamma\left(1-\delta_{1}\right)}+2-\Delta_{1}\right] \sum_{i=1}^{n} M_{i} \cdot\|x\|_{\mathrm{PC}} \\
& \leq\left[\left(L_{1}+L_{2}\right)\left(\frac{1}{\Gamma(\alpha+1)}+\frac{1}{\left(1-\Delta_{1}\right) \Gamma\left(\alpha-\delta_{1}+1\right)}+\frac{1}{\left(1-\Delta_{1}\right) \Gamma(\alpha+1)}\right)\right. \\
& \left.+\frac{\Gamma\left(2-\gamma_{1}\right)}{1-\Delta_{1}}\left(\frac{2}{t_{1}^{1-\gamma_{1}}}+\frac{1}{z^{\delta_{1}} \Gamma\left(1-\delta_{1}\right)}+2-\Delta_{1}\right) \sum_{i=1}^{n} M_{i}\right]\|x\|_{\mathrm{PC}} \\
& +N\left(L_{1}+L_{2}\right)\left(\frac{1}{\Gamma(\alpha+1)}+\frac{1}{\left(1-\Delta_{1}\right) \Gamma\left(\alpha-\delta_{1}+1\right)}+\frac{1}{\left(1-\Delta_{1}\right) \Gamma(\alpha+1)}\right) \\
& \leq\left(\mathcal{M}_{1}+\mathcal{N}_{1}\right) r+N \mathcal{M}_{1}=\kappa_{1} r+N \mathcal{M}_{1} \leq r .
\end{aligned}
$$

Similarly, we also have

$$
\left|T_{2}(x, y)(t)\right| \leq \kappa_{2} r+\hat{N} \mathcal{M}_{2} \leq r
$$

Estimates (3.6) and (3.7) indicate that $T$ is uniformly bounded and $T(\bar{\Omega}) \subset \bar{\Omega}$.

Next, we show that operator $T$ is equicontinuous, that is, for any $\epsilon>0, \tau_{2}, \tau_{1} \in J=[0,1]$, $(x, y) \in \bar{\Omega}$, there exists $\delta=\delta(\epsilon)>0$ such that, when $\left|\tau_{2}-\tau_{1}\right|<\delta$, we have $\| T(x, y)\left(\tau_{2}\right)-$ $T(x, y)\left(\tau_{1}\right) \|<\epsilon$. Indeed, for any $\tau_{1}, \tau_{2} \in[0,1]$, without loss of generality, let $\tau_{1}<\tau_{2}$ and $\left|\tau_{2}-\tau_{1}\right|<\xi$, where $\xi=\min _{0 \leq i \leq n}\left\{t_{i+1}-t_{i}\right\}, t_{0}=0, t_{n+1}=1$. Similar to (3.6), we have

$$
\begin{aligned}
& \left|T_{1}(x, y)\left(\tau_{2}\right)-T_{1}(x, y)\left(\tau_{1}\right)\right| \\
& \quad \leq\left|I_{0^{+}}^{\alpha} f\left(\tau_{2}, x\left(\tau_{2}\right),{ }^{C} D_{0^{+}}^{p} y\left(\tau_{2}\right)\right)-I_{0^{+}}^{\alpha} f\left(\tau_{1}, x\left(\tau_{1}\right),{ }^{C} D_{0^{+}}^{p} y\left(\tau_{1}\right)\right)\right| \\
& \quad+\frac{I_{0^{+}}^{\alpha-\delta_{1}}\left|f\left(z, x(z),{ }^{C} D_{0^{+}}^{p} y(z)\right)\right|+I_{0^{+}}^{\alpha}\left|f\left(1, x(1),{ }^{C} D_{0^{+}}^{p} y(1)\right)\right|}{1-\Delta_{1}}\left|\tau_{2}-\tau_{1}\right|
\end{aligned}
$$




$$
\begin{aligned}
& +\left[\frac{\left|C_{1}\right|}{1-\Delta_{1}}+\sum_{i=1}^{k} \frac{\Gamma\left(2-\gamma_{1}\right)}{t_{i}^{1-\gamma_{1}}}\left|J_{1 i}\left(x\left(t_{i}\right)\right)\right|\right]\left|\tau_{2}-\tau_{1}\right| \\
& \leq \frac{1}{\Gamma(\alpha)} \int_{0}^{\tau_{1}}\left[\left(\tau_{2}-s\right)^{\alpha-1}-\left(\tau_{1}-s\right)^{\alpha-1}\right]\left|f\left(s, x(s),{ }^{C} D_{0^{+}}^{p} y(s)\right)\right| d s \\
& +\frac{1}{\Gamma(\alpha)} \int_{\tau_{1}}^{\tau_{2}}\left(\tau_{2}-s\right)^{\alpha-1}\left|f\left(s, x(s),{ }^{C} D_{0^{+}}^{p} y(s)\right)\right| d s \\
& +\frac{I_{0^{+}}^{\alpha-\delta_{1}}\left|f\left(z, x(z),{ }^{C} D_{0^{+}}^{p} y(z)\right)\right|+I_{0^{+}}^{\alpha}\left|f\left(1, x(1),{ }^{C} D_{0^{+}}^{p} y(1)\right)\right|}{1-\Delta_{1}}\left|\tau_{2}-\tau_{1}\right| \\
& +\left[\frac{\left|C_{1}\right|}{1-\Delta_{1}}+\sum_{i=1}^{k} \frac{\Gamma\left(2-\gamma_{1}\right)}{t_{i}^{1-\gamma_{1}}}\left|J_{1 i}\left(x\left(t_{i}\right)\right)\right|\right]\left|\tau_{2}-\tau_{1}\right| \\
& \leq \frac{1}{\Gamma(\alpha+1)}\left(L_{1}\|x\|_{\mathrm{PC}}+L_{2}\|y\|_{\mathrm{PC}}+N\right)\left(\tau_{2}^{\alpha}-\tau_{1}^{\alpha}-\left(\tau_{2}-\tau_{1}\right)^{\alpha}\right) \\
& +\frac{1}{\Gamma(\alpha+1)}\left(L_{1}\|x\|_{\mathrm{PC}}+L_{2}\|y\|_{\mathrm{PC}}+N\right)\left(\tau_{2}-\tau_{1}\right)^{\alpha} \\
& +\frac{1}{\left(1-\Delta_{1}\right) \Gamma\left(\alpha-\delta_{1}+1\right)}\left(L_{1}\|x\|_{\mathrm{PC}}+L_{2}\|y\|_{\mathrm{PC}}+N\right)\left|\tau_{2}-\tau_{1}\right| \\
& +\frac{1}{\left(1-\Delta_{1}\right) \Gamma(\alpha+1)}\left(L_{1}\|x\|_{\mathrm{PC}}+L_{2}\|y\|_{\mathrm{PC}}+N\right)\left|\tau_{2}-\tau_{1}\right| \\
& +\frac{\Gamma\left(2-\gamma_{1}\right)}{1-\Delta_{1}}\left[\frac{2}{t_{1}^{1-\gamma_{1}}}+\frac{1}{z^{\delta_{1}} \Gamma\left(1-\delta_{1}\right)}+1\right] \sum_{i=1}^{n} M_{i} \cdot\|x\|_{\mathrm{PC}} \cdot\left|\tau_{2}-\tau_{1}\right| \\
& \leq \frac{1}{\Gamma(\alpha+1)}\left(L_{1} r+L_{2} r+N\right)\left(\tau_{2}^{\alpha}-\tau_{1}^{\alpha}\right) \\
& +\frac{1}{\left(1-\Delta_{1}\right) \Gamma\left(\alpha-\delta_{1}+1\right)}\left(L_{1} r+L_{2} r+N\right)\left|\tau_{2}-\tau_{1}\right| \\
& +\frac{1}{\left(1-\Delta_{1}\right) \Gamma(\alpha+1)}\left(L_{1} r+L_{2} r+N\right)\left|\tau_{2}-\tau_{1}\right| \\
& +\frac{\Gamma\left(2-\gamma_{1}\right)}{1-\Delta_{1}}\left[\frac{2}{t_{1}^{1-\gamma_{1}}}+\frac{1}{z^{\delta_{1}} \Gamma\left(1-\delta_{1}\right)}+1\right] \sum_{i=1}^{n} M_{i} \cdot r \cdot\left|\tau_{2}-\tau_{1}\right| \\
& =\frac{1}{\Gamma(\alpha)}\left(L_{1} r+L_{2} r+N\right) \eta^{\alpha-1}\left|\tau_{2}-\tau_{1}\right| \\
& +\frac{1}{\left(1-\Delta_{1}\right) \Gamma\left(\alpha-\delta_{1}+1\right)}\left(L_{1} r+L_{2} r+N\right)\left|\tau_{2}-\tau_{1}\right| \\
& +\frac{1}{\left(1-\Delta_{1}\right) \Gamma(\alpha+1)}\left(L_{1} r+L_{2} r+N\right)\left|\tau_{2}-\tau_{1}\right| \\
& +\frac{\Gamma\left(2-\gamma_{1}\right)}{1-\Delta_{1}}\left[\frac{2}{t_{1}^{1-\gamma_{1}}}+\frac{1}{z^{\delta_{1}} \Gamma\left(1-\delta_{1}\right)}+1\right] \sum_{i=1}^{n} M_{i} \cdot r \cdot\left|\tau_{2}-\tau_{1}\right| \\
& \leq\left[\left(L_{1} r+L_{2} r+N\right)\left(\frac{1}{\Gamma(\alpha)}+\frac{1}{\left(1-\Delta_{1}\right) \Gamma\left(\alpha-\delta_{1}+1\right)}+\frac{1}{\left(1-\Delta_{1}\right) \Gamma(\alpha+1)}\right)\right. \\
& \left.+\frac{r \Gamma\left(2-\gamma_{1}\right)}{1-\Delta_{1}}\left(\frac{2}{t_{1}^{1-\gamma_{1}}}+\frac{1}{z^{\delta_{1}} \Gamma\left(1-\delta_{1}\right)}+1\right) \sum_{i=1}^{n} M_{i}\right]\left|\tau_{2}-\tau_{1}\right| \\
& =\rho_{1}\left|\tau_{2}-\tau_{1}\right|,
\end{aligned}
$$


where $\tau_{1}<\eta<\tau_{2}, \rho_{1}=\left(L_{1} r+L_{2} r+N\right)\left(\frac{1}{\Gamma(\alpha)}+\frac{1}{\left(1-\Delta_{1}\right) \Gamma\left(\alpha-\delta_{1}+1\right)}+\frac{1}{\left(1-\Delta_{1}\right) \Gamma(\alpha+1)}\right)+\frac{r \Gamma\left(2-\gamma_{1}\right)}{1-\Delta_{1}}\left(\frac{2}{t_{1}^{1-\gamma_{1}}}+\right.$ $\left.\frac{1}{z^{\delta_{1}} \Gamma\left(1-\delta_{1}\right)}+1\right) \sum_{i=1}^{n} M_{i}$. Similar to (3.8), one has

$$
\left|T_{2}(x, y)\left(\tau_{2}\right)-T_{1}(x, y)\left(\tau_{1}\right)\right| \leq \rho_{2}\left|\tau_{2}-\tau_{1}\right|
$$

where $\rho_{2}=\left(\hat{L}_{1} r+\hat{L}_{2} r+\hat{N}\right)\left(\frac{1}{\Gamma(\beta)}+\frac{1}{\left(1-\Delta_{2}\right) \Gamma\left(\beta-\delta_{2}+1\right)}+\frac{1}{\left(1-\Delta_{2}\right) \Gamma(\beta+1)}\right)+\frac{r \Gamma\left(2-\gamma_{2}\right)}{1-\Delta_{2}}\left(\frac{2}{t_{1}^{1-\gamma_{2}}}+\frac{1}{w^{\delta_{2}} \Gamma\left(1-\delta_{2}\right)}+\right.$ 1) $\sum_{i=1}^{n} \hat{M}_{i}$.

Take $\delta=\min \left\{\xi, \frac{\epsilon}{\rho_{1}}, \frac{\epsilon}{\rho_{1}}\right\}$. According to (3.8) and (3.9), we conclude that, for any $\epsilon>0$, $\tau_{2}, \tau_{1} \in J=[0,1],(x, y) \in \bar{\Omega}$, there exists $\delta>0$ such that $\left\|T(x, y)\left(\tau_{2}\right)-T(x, y)\left(\tau_{1}\right)\right\|<\epsilon$ if $\left|\tau_{2}-\tau_{1}\right|<\delta$, namely, operator $T$ is equicontinuous. Hence, by the Arzela-Ascoli theorem, we know that $T: \bar{\Omega} \rightarrow \bar{\Omega}$ is completely continuous.

Finally, we prove that condition (ii) of Lemma 2.4 is not true. In fact, for all $(\bar{x}, \bar{y}) \in \partial \Omega$, $0<\lambda<1$ and $t \in[0,1]$, analogous to (3.6) and (3.7), we have

$$
\left|\lambda T_{1}(\bar{x}, \bar{y})(t)\right| \leq \lambda\left(\kappa_{1}\|\bar{x}\|_{\mathrm{PC}}+N \mathcal{M}_{1}\right)<\|(\bar{x}, \bar{y})\|=r
$$

and

$$
\left|\lambda T_{2}(\bar{x}, \bar{y})(t)\right| \leq \lambda\left(\kappa_{2}\|\bar{x}\|_{\mathrm{PC}}+\hat{N} \mathcal{M}_{2}\right)<\|(\bar{x}, \bar{y})\|=r
$$

Estimates (3.10) and (3.11) imply that $\|\lambda T(\bar{x}, \bar{y})\|<\|(\bar{x}, \bar{y})\|=r$, that is, $(\bar{x}, \bar{y}) \neq \lambda T(\bar{x}, \bar{y})$, for all $(\bar{x}, \bar{y}) \in \partial \Omega$. According to Lemma 2.4, we know that the boundary value problem (1.1) has a pair of solutions $\left(x^{*}, y^{*}\right) \in \bar{\Omega}$. The proof is completed.

\section{Illustrative examples}

Consider the following four-point boundary value problem for nonlinear fractional differential coupling system with fractional order impulses:

$$
\left\{\begin{array}{l}
{ }^{C} D_{0^{+}}^{\alpha} x(t)=f\left(t, x(t),{ }^{C} D_{0^{+}}^{p} y(t)\right), \quad t \in J=[0,1], t \neq t_{k}, \\
{ }^{C} D_{0^{+}}^{\beta} y(t)=g\left(t,{ }^{C} D_{0^{+}}^{q} x(t), y(t)\right), \quad t \in J=[0,1], t \neq t_{k}, \\
{ }^{C} D_{0^{+}}^{\gamma_{1}} x\left(t_{k}^{+}\right)-{ }^{C} D_{0^{+}}^{\gamma_{1}} x\left(t_{k}^{-}\right)=J_{1 k}\left(x\left(t_{k}\right)\right), \quad k=1, \ldots, n, \\
{ }^{C} D_{0^{+}}^{\gamma_{2}} y\left(t_{k}^{+}\right)-{ }^{C} D_{0^{+}}^{\gamma_{2}} y\left(t_{k}^{-}\right)=J_{2 k}\left(y\left(t_{k}\right)\right), \quad k=1, \ldots, n, \\
x(0)=y(0)=0, \quad{ }^{\mathrm{LR}} D_{0^{+}}^{\delta_{1}} x(z)=x(1), \quad{ }^{\mathrm{LR}} D_{0^{+}}^{\delta_{2}} y(w)=y(1) .
\end{array}\right.
$$

Take $\alpha=\frac{5}{4}, \beta=\frac{7}{4}, p=\frac{1}{2}, q=\frac{3}{4}, \gamma_{1}=\frac{1}{3}, \gamma_{2}=\frac{2}{3}, \delta_{1}=\frac{1}{5}, \delta_{2}=\frac{3}{5}, n=2, t_{1}=\frac{1}{6}, t_{2}=\frac{5}{6}, z=\frac{1}{7}, w=$ $\frac{4}{7}, f(t, u, v)=\frac{\sin (\pi t)+u+v}{100}, g(t, u, v)=\frac{e^{t}+\arctan \left(u^{2}+v^{2}\right)}{100}, J_{11}(u)=J_{22}(u)=\frac{u^{2}}{200}, J_{12}(u)=J_{21}(u)=\frac{\sqrt[3]{u}}{100}$. Obviously, $f, g \in C\left(J \times R^{2}, R\right), J_{11}, J_{12}, J_{21}, J_{22} \in C(R, R)$. By a simple calculation, we have

$$
\begin{aligned}
& \left|f\left(t, u_{1}, v_{1}\right)-f\left(t, u_{2}, v_{2}\right)\right| \leq \frac{1}{100}\left|u_{1}-u_{2}\right|+\frac{1}{100}\left|v_{1}-v_{2}\right|, \\
& \left|g\left(t, u_{1}, v_{1}\right)-g\left(t, u_{2}, v_{2}\right)\right| \leq \frac{1}{100}\left|u_{1}-u_{2}\right|+\frac{1}{100}\left|v_{1}-v_{2}\right|, \\
& \left|J_{11}(u)\right|=\left|J_{22}(u)\right| \leq \frac{1}{50}|u|, \quad\left|J_{12}(u)\right|=\left|J_{21}(u)\right| \leq \frac{1}{300}|u|, \\
& \sup _{t \in[0,1]}|f(t, 0,0)|=\frac{1}{100}, \quad \sup _{t \in[0,1]}|g(t, 0,0)|=\frac{e}{100},
\end{aligned}
$$


that is, $L_{1}=L_{2}=\hat{L}_{1}=\hat{L}_{2}=\frac{1}{100}, M_{1}=\hat{M}_{2}=\frac{1}{100}, M_{2}=\hat{M}_{1}=\frac{1}{300}, N=\frac{1}{100}, \hat{N}=\frac{e}{100}$. Therefore, we obtain

$$
\begin{aligned}
& 0<\Delta_{1}=\frac{z^{1-\delta_{1}}}{\Gamma\left(2-\delta_{1}\right)} \approx 0.2264<1, \quad 0<\Delta_{2}=\frac{w^{1-\delta_{2}}}{\Gamma\left(2-\delta_{2}\right)} \approx 0.9010<1, \\
& \mathcal{M}_{1}=\left(L_{1}+L_{2}\right)\left(\frac{1}{\Gamma(\alpha+1)}+\frac{1}{\left(1-\Delta_{1}\right) \Gamma\left(\alpha-\delta_{1}+1\right)}+\frac{1}{\left(1-\Delta_{1}\right) \Gamma(\alpha+1)}\right) \approx 0.0658, \\
& \mathcal{N}_{1}=\frac{\Gamma\left(2-\gamma_{1}\right)}{1-\Delta_{1}}\left(\frac{2}{t_{1}^{1-\gamma_{1}}}+\frac{1}{z^{\delta_{1}} \Gamma\left(1-\delta_{1}\right)}+2-\Delta_{1}\right) \sum_{i=1}^{n} M_{i} \approx 0.1501, \\
& \mathcal{M}_{2}=\left(\hat{L}_{1}+\hat{L}_{2}\right)\left(\frac{1}{\Gamma(\beta+1)}+\frac{1}{\left(1-\Delta_{2}\right) \Gamma\left(\beta-\delta_{2}+1\right)}+\frac{1}{\left(1-\Delta_{2}\right) \Gamma(\beta+1)}\right) \approx 0.3263, \\
& \mathcal{N}_{2}=\frac{\Gamma\left(2-\gamma_{2}\right)}{1-\Delta_{2}}\left(\frac{2}{t_{1}^{1-\gamma_{2}}}+\frac{1}{w^{\delta_{2}} \Gamma\left(1-\delta_{2}\right)}+2-\Delta_{2}\right) \sum_{i=1}^{n} \hat{M}_{i} \approx 0.6451, \\
& \kappa_{1}=\mathcal{M}_{1}+\mathcal{N}_{1} \approx 0.2159<1, \quad \kappa_{2}=\mathcal{M}_{2}+\mathcal{N}_{2} \approx 0.9714<1 .
\end{aligned}
$$

Thus, conditions $\left(H_{1}\right)-\left(H_{6}\right)$ of Theorem 3.1 hold. Then (4.1) has at least a pair of solutions.

\section{Conclusions}

In describing some phenomena and processes of many fields such as physics, chemistry, aerodynamics, electrodynamics of a complex medium, polymer rheology, capacitor theory, electrical circuits, biology, control theory, fitting of experimental data, and so on, the fractional differential equation is better and more accurate than the integral-order differential equations. So the study of fractional differential equations has attracted the eyes of many scholars. Especially, the nonlocal boundary value problems have been widely studied by many researchers because of their extensive applications in, e.g., blood flow problems, chemical engineering, thermo-elasticity, underground water flow, population dynamics, and so forth. In this paper, we consider the nonlocal boundary value problem for a nonlinear fractional differential coupled system with fractional order impulses. We obtain some new sufficient criteria for the existence of solutions by use of the Leray-Schauder alternative theorem.

Acknowledgements

The authors thank the referees for a number of suggestions which have improved many aspects of this article.

Funding

This work was supported by the National Natural Sciences Foundation of Peoples Republic of China under Grant (Nos. 11161025, 11661047).

Availability of data and materials

Not applicable.

Competing interests

The authors declare to have no competing interests.

Authors' contributions

The authors read and approved the final manuscript.

Publisher's Note

Springer Nature remains neutral with regard to jurisdictional claims in published maps and institutional affiliations. 


\section{References}

1. Li, X., Chen, F., Li, X.: Generalized anti-periodic boundary value problems of impulsive fractional differential equations. Commun. Nonlinear Sci. Numer. Simul. 18, 28-41 (2013)

2. Ahmad, B., Nieto, J.: Existence results for nonlinear boundary value problems of fractional integrodifferential equations with integral boundary conditions. Bound. Value Probl. 2009, Article ID 708576 (2009)

3. Ahmad, B.: Nonlinear fractional differential equations with anti-periodic type fractional boundary conditions. Differ. Equ. Dyn. Syst. 21(4), 387-401 (2013)

4. Zhao, K., Gong, P.: Existence of positive solutions for a class of higher-order Caputo fractional differential equation. Qual. Theory Dyn. Syst. 14(1), 157-171 (2015)

5. Guezane-Lakoud, A., Khaldi, R.: Solvability of a fractional boundary value problem with fractional integral condition. Nonlinear Anal. 75, 2692-2700 (2012)

6. Zhao, K.: Triple positive solutions for two classes of delayed nonlinear fractional FDEs with nonlinear integral boundary value conditions. Bound. Value Probl. 2015, 181 (2015)

7. Wang, J., LV, L., Zhou, Y.: Boundary value problems for fractional differential equations involving Caputo derivative in Banach spaces. J. Appl. Math. Comput. 38, 209-224 (2012)

8. Zhao, K., Gong, P.: Positive solutions of Riemann-Stieltjes integral boundary problems for the nonlinear coupling system involving fractional-order differential. Adv. Differ. Equ. 2014, 254 (2014)

9. Zhao, K., Wang, K.: Existence of solutions for the delayed nonlinear fractional functional differential equations with three-point integral boundary value conditions. Adv. Differ. Equ. 2016, 284 (2016)

10. Ahmad, B., Alsaedi, A.: Nonlinear fractional differential equations with nonlocal fractional integro-differential boundary conditions. Bound. Value Probl. 2012, Article ID 691721 (2012)

11. Zhao, K., Gong, P.: Positive solutions of $m$-point multi-term fractional integral BVP involving time-delay for fractional differential equations. Bound. Value Probl. 2015, 19 (2015)

12. Zhang, Y., Bai, Z., Feng, T.: Existence results for a coupled system of nonlinear fractional three-point boundary value problems at resonance. Comput. Math. Appl. 61, 1032-1047 (2011)

13. Ahmad, B., Sotiris, K., Ahmed, A.: On a coupled system of fractional differential equations with coupled nonlocal and integral boundary conditions. Chaos Solitons Fractals 83, 234-241 (2016)

14. Zhao, K., Gong, P.: Positive solutions of nonlocal integral BVPs for the nonlinear coupled system involving high-order fractional differential. Math. Slovaca 67(2), 447-466 (2017)

15. Shu, X., Lai, Y., Chen, Y.: The existence of mild solutions for impulsive fractional partial differential equations. Nonlinear Anal., Theory Methods Appl. 74(5), 2003-2011 (2011)

16. Wang, J., Zhou, Y., Fĕckan, M.: On recent developments in the theory of boundary value problem for impulsive fractional differential equations. Comput. Math. Appl. 64(10), 3008-3020 (2012)

17. Tian, Y., Ge, W.: Variational methods to Sturm-Liouville boundary value problem for impulsive differential equations. Nonlinear Anal., Theory Methods Appl. 72, 277-287 (2010)

18. Zhao, K., Gong, P.: Positive solutions for impulsive fractional differential equations with generalized periodic boundary value conditions. Adv. Differ. Equ. 2014, 255 (2014)

19. Agarwal, R., Benchohra, M., Slimani, B.: Existence results for differential equations with fractional order and impulses. Mem. Differ. Equ. Math. Phys. 44, 1-21 (2008)

20. Zhao, K.: Impulsive boundary value problems for two classes of fractional differential equation with two different Caputo fractional derivatives. Mediterr. J. Math. 13, 1033-1050 (2016)

21. Ahmad, B., Sivasundaram, S.: Existence of solutions for impulsive integral boundary value problems of fractional order. Nonlinear Anal. Hybrid Syst. 4, 134-141 (2010)

22. Benchohra, M., Slimani, B.: Existence and uniqueness of solutions to impulsive fractional differential equations. Electron. J. Differ. Equ. 2009, Article ID 10 (2009)

23. Zhao, K.: Multiple positive solutions of integral BVPs for high-order nonlinear fractional differential equations with impulses and distributed delays. Dyn. Syst. 30(2), 208-223 (2015)

24. Zhao, K., Suo, L.: Solvability of nonlocal boundary value problem for a class of nonlinear fractional differential coupled system with impulse. Adv. Differ. Equ. 2018, 21 (2018)

25. Zhao, K., Liang, J.: Solvability of triple-point integral boundary value problems for a class of impulsive fractional differential equations. Adv. Differ. Equ. 2017, 50 (2017)

26. Zhang, X., Zhu, C., Wu, Z:: Solvability for a coupled system of fractional differential equations with impulses at resonance. Bound. Value Probl. 2013, 80 (2013)

27. Song, Q., Bai, Z.: Positive solutions of fractional differential equations involving the Riemann-Stieltjes integral boundary condition. Adv. Differ. Equ. 2018, 183 (2018)

28. Bai, Z., Chen, Y., Lian, H., Sun, S.: On the existence of blow up solutions for a class of fractional differential equations. Fract. Calc. Appl. Anal. 17(4), 1175-1187 (2014)

29. Sheng, K., Zhang, W., Bai, Z.: Positive solutions to fractional boundary-value problems with p-Laplacian on time scales. Bound. Value Probl. 2018, 70 (2018)

30. Tian, Y., Bai, Z.: Existence results for the three-point impulsive boundary value problem involving fractional differential equations. Comput. Math. Appl. 59(8), 2601-2609 (2010)

31. Zhang, Y.: Existence results for a coupled system of nonlinear fractional multi-point boundary value problems at resonance. J. Inequal. Appl. 2018, 198 (2018)

32. Kilbas, A., Srivastava, H., Trujillo, J.: Theory and Applications of Fractional Differential Equations. North-Holland Mathematics Studies, vol. 204. Elsevier, Amsterdam (2006)

33. Podlubny, I.: Fractional Differential Equations. Academic Press, New York (1993)

34. Diethelm, K.: The Analysis of Fractional Differential Equations. Springer, Berlin (2010)

35. Zeidler, E.: Nonlinear Functional Analysis and Applications I: Fixed Point Theorems. Springer, New York (1986) 